\title{
Mutations in $A R$ or SRD5A2 Genes: Clinical Findings, Endocrine Pitfalls, and Genetic Features of Children with 46,XY DSD
}

\author{
(D) Neşe Akcan1, (D) Oya Uyguner2, (I) Firdevs Baş3, (D) Umut Altunoğlu2,4, (D) Güven Toksoy², (D) Birsen Karaman², (D) Şahin Avcı2,4, \\ (D) Zehra Yavaş Abalı3 , (D) Şükran Poyrazoğlu33, (1) Agharza Aghayev², (D) Volkan Karaman², (D) Rüveyde Bundak5 , (D) Seher Başaran², \\ (D) Feyza Darendeliler 3
}

${ }^{1}$ Near East University Faculty of Medicine, Department of Pediatric Endocrinology, Nicosia, Cyprus 2istanbul University, Istanbul Faculty of Medicine, Department of Medical Genetics, Istanbul, Turkey

3istanbul University, Istanbul Faculty of Medicine, Department of Pediatric Endocrinology, İstanbul, Turkey

${ }^{4}$ Koç University Faculty of Medicine, Department of Medical Genetics, Istanbul, Turkey

5 University of Kyrenia, Faculty of Medicine, Department of Pediatric Endocrinology, Kyrenia, Cyprus

\begin{abstract}
What is already known on this topic?
Androgen insensivity syndrome and $5 \alpha$-reductase deficiency are the most common causes of 46 ,XY disorders of sexual development. They can present as indistinguishable phenotypes that usually necessitate molecular analyses for the definitive diagnosis in the prepubertal period.
\end{abstract}

\section{What this study adds?}

Testosterone to dihydrotestosterone ratio may lead to diagnostic confusion. Genetic analysis for actual diagnosis seems to be essential. Four novel androgen receptor variants were identified in this Turkish pediatric population.

\begin{abstract}
Objective: Androgen insensivity syndrome (AIS) and $5 \alpha$-reductase deficiency ( $5 \alpha-$ RD) present with indistinguishable phenotypes among the 46,XY disorders of sexual development (DSD) that usually necessitate molecular analyses for the definitive diagnosis in the prepubertal period. The aim was to evaluate the clinical, hormonal and genetic findings of 46,XY DSD patients who were diagnosed as AIS or $5 \alpha-R D$.

Methods: Patients diagnosed as AIS or $5 \alpha-R D$ according to clinical and hormonal evaluations were investigated. Sequence variants of steroid 5- $\alpha$-reductase type 2 were analyzed in cases with testosterone/dihydrotestosterone (T/DHT) ratio of $\geq 20$, whereas the androgen receptor $(A R)$ gene was screened when the ratio was $<20$. Stepwise analysis of other associated genes were screened in cases with no causative variant found in initial analysis. For statistical comparisons, the group was divided into three main groups and subgroups according to their genetic diagnosis and T/DHT ratios.

Results: A total of 128 DSD patients from 125 non-related families were enrolled. Birth weight SDS and gestational weeks were significantly higher in $5 \alpha$-RD group than in AIS and undiagnosed groups. Completely female phenotype was higher in all subgroups of both AIS and $5 \alpha-$ RD patients than in the undiagnosed subgroups. In those patients with stimulated T/DHT $<20$ in the prepubertal period, stimulated T/DHT ratio was significantly lower in AIS than in the undiagnosed group, and higher in $5 \alpha$-RD. Phenotype associated variants were detected in $24 \%$ ( $n=18$ AIS, $n=145 \alpha-R D)$ of the patients, revealing four novel $A R$ variants (c.94G > T, p.Glu32* c.330G > C, p.Leu110 = ; c.2084C > T, p.Pro695Leu, c.2585_2592delAGCTCCTG, p.(Lys862Argfs*16), of these c.330G > C with silent status remained undefined in terms of its causative effects.

Conclusion: T/DHT ratio is an important hormonal criterion, but in some cases, T/DHT ratio may lead to diagnostic confusion. Molecular diagnosis is important for the robust diagnosis of 46,XY DSD patients. Four novel $A R$ variants were identified in our study.

Keywords: 46,XY disorders of sex development, $5 \alpha$-reductase deficiency, androgen insensitivity syndrome, androgen receptor gene mutations, SRD5A2 gene mutations
\end{abstract}

Address for Correspondence: Neşe Akcan MD, Near East University Faculty of Medicine, Department of Pediatric Conflict of interest: None declared Endocrinology, Nicosia, Cyprus

Phone: + 903926751000 (1388) E-mail: nese.akcan@med.neu.edu.tr ORCID: orcid.org/0000-0003-2583-5736

${ }^{\circ}$ Copyright 2022 by Turkish Society for Pediatric Endocrinology and Diabetes

The Journal of Clinical Research in Pediatric Endocrinology published by Galenos Publishing House. 


\section{Introduction}

Acompletelyvirilized male phenotype requires a 46, XY chromosome, adequate testosterone ( $\mathrm{T}$ ) and dihydrotestosterone (DHT) which is formed from $\mathrm{T}$ by the enzyme steroid 5- $\alpha$-reductase type 2 (SRD5A2) and, fully functionally active androgen receptors (AR) and post receptor pathways $(1,2,3)$. A disturbance at any of those last stages can lead to 46,XY disorders of sex development (DSD), characterized by a range of female phenotype to incompletely virilized external genitalia $(1,2,3)$. Although a significant proportion of $46, X Y$ DSD cases may remain aetiologically unclarified, the most common identifiable cause in the reported series is androgen insensitivity syndrome (AIS) and $5 \alpha$-reductase deficiency $(5 \alpha-\mathrm{RD})$ is the second most common cause $(1,2,3)$. The diagnosis of AIS requires the exclusion of other aetiologies of $46, \mathrm{XY}$ DSD, which are gonadal differentiation defects, and $\mathrm{T}$ biosynthesis and metabolism. In the prepubertal period, phenotypes of AIS, $5 \alpha$-RDor unknown aetiologies of 46 ,XY DSD are indistinguishable because of the similarities intheir clinical findings. Although adequate serum $\mathrm{T}$ concentrations rule out a defect in $\mathrm{T}$ biosynthesis, a low $\mathrm{T}$ value at baseline or following human chorionic gonadotrophin (hCG) stimulation does not always rule out AIS (2). On the other hand, phenotype at birth varies widely in $5 \alpha-\mathrm{RD}$, according to the levels of residual enzymatic function, so a very similar clinical picture to AIS at prepubertal ages with normal testicular $\mathrm{T}$ production can be observed in these patients. Although serum T/DHT ratio is an important screening tool for identifying patients with possible $5 \alpha-R D$, cut-off values for diagnosis remain uncertain. Cases with a molecular diagnosis of $5 \alpha-R D$ and T/DHT ratio below the suggested cut-off values have also been reported (4). So, there are overlaps in the diagnosis of AIS or $5 \alpha-R D$ based on clinical or hormonal data and to distinguish them from each other or to differentiate these two main or most common diagnoses from unknown aetiologies, molecular analyses is necessary.

The $A R$ gene is located on the X-chromosome inthe $\mathrm{Xq} 12$ region, composed of eight exons and encodes a protein with 920 aa in length (NP_000035), that function as a transcription factoractivated via binding of steroid hormones. The peptide chain of AR consists of three domains:residues at the $\mathrm{N}$-terminal region between 6-449 aa encodes androgen receptor domain (NTD; N terminal domain); 558-627 aa zinc finger $\mathrm{C} 4$ type domain (DBD, DNA binding domain); and 690-881 ligand binding domain (LBD) of nuclear hormone receptor (EMBL-EBI; P10275, Pfam) (PubMed: 16381856) (5,6). Loss-of-function mutation of $A R$ is responsible for $\mathrm{X}$-linked androgen insensitivity
(MIM\#300068) and hypospadias (MIM\# 300633) in humans. The SRD5A2 gene is located at 2p23, comprises five exons encoding a 254 aa peptide chain that encircles 3-oxo-5-alpha-steroid 4-dehydrogenase domain, encoded by residues between 105-254 (EMBL-EBI; P31213, Pfam) (PubMed: 16381856). Bi-allellic pathogenic alterations of SRD5A2 are associated with pseudovaginal perineoscrotal hypospadias (MIM\# 264600) caused by steroid $5 \alpha-R D$.

Since previous studies have indicated $A R$ and SRD5A2 gene mutationsas the most common culprits behind 46,XY DSD for the most part $(1,2,3)$, we focused on these two genes in patients with 46,XY DSDs with normal testicular development. Here, a relatively large cohort of Turkish children is reported to present the clinical, hormonal and genetic features of 46,XY DSD patients who were considered as AIS or $5 \alpha-R D$ and to analyze the accordance between the clinical and laboratory results withgenetic analysis.

\section{Methods}

\section{Participants}

A retrospective medical chart review of 46,XY DSD patients was performed to collect data from the Pediatric Endocrinology Outpatient Clinic of İstanbul University, İstanbul, Turkey. DSD patients who were diagnosed as AIS or $5 \alpha-\mathrm{RD}$ according to the clinical, hormonal or molecular evalutions, were included in the study. Since the study was performed respectively, patient-informed consent forms were not needed. The study has been reviewed by the Ethics Committee of İstanbul Faculty of Medicine, İstanbul University, and has therefore been performed in accordance with the ethical standards laid down in an appropriate version of the Declaration of Helsinki. Initially, we sought all cases with AIS, $5 \alpha-R D$ or undiagnosed groups according to their molecular diagnosis. In addition, the participants were also subclassified according to their T/DHT for statistical analysis.Clinical diagnosis of AIS or $5 \alpha-R D$ was based on normal $\mathrm{T}$ secretion without Mullerian duct structures. Criteria suggesting DSD included overt genital ambiguity, apparent female genitalia with or without clitoromegaly, posterior labial fusion or inguinal/labial mass, and apparent male genitalia with non-palpable testes, micropenis, isolated perineal hypospadias or mild hypospadias with undescended testis (7). Also, file records of older children and adolescents who hadincomplete or delayed puberty, lack of breast development or primary amenorrhea or virilization at puberty were retrospectively evaluated with respect to DSD. A detailed history including age at presentation, main complaints, sex of rearing, and parental consanguinity were recorded for each patient. A clinical examination 
consisting of anthropometry, assessment of pubertal stage, severity of ambigious genitalia, penile length and associated anomalies or dysmorphic features were evaluated for each patient. Quigley scale for grading AIS was used to determine the degree of external virilization in $46, \mathrm{XY}$ DSD patients $(8,9,10)$. Grades 2 through 5 quantify four degrees of increasingly feminized genitalia that correspond to partial AIS (PAIS). Grades 1 and 6/7 correspond to mild AIS (MAIS) and complete AIS (CAIS), respectively (10). The external masculinization score (EMS, range 0-12) was also assessed in patients $(11,12)$. The standard deviation score (SDS) of height and weight were calculated according to the reported data of Neyzi et al (13) for Turkish children and adolescence, whereas birth weight SDS based on gestation week, were calculated according to reported national data for Turkish newborns (14). Patients were subdivided into three groups according to birth weight SDS: small for gestational age [SGA (<-2 SDS)], large for gestational age [LGA (> + 2 SDS)] and appropriate for gestational age [AGA (between -2 and +2 SDS)]

\section{Laboratory Monitoring}

As a part of routine evaluation of DSD, we performed hormonal measurements, karyotype analysis, abdominopelvic and scrotal ultrasound and, if required, magnetic resonance imaging. Basal level of T, DHT, T/DHT ratio and gonadotropins were measured at pubertal age or in mini puberty. A short-term hCG test was applied in the appropriate cases who were in the prepubertal period. The hCG stimulation test was carried out by administering $1500 \mathrm{IU} / \mathrm{m}^{2} /$ dose of hCG daily IM for three consecutive days to determine the ability of the gonads to produce $\mathrm{T}$ and DHT. Blood samples were obtained before the first dose and $24 \mathrm{~h}$ after the last (15). An increment in plasma $\mathrm{T}(\Delta \mathrm{T})$ of more than $0.8 \mathrm{ng} / \mathrm{mL}$ or an absolute level greater than $0.9 \mathrm{ng} / \mathrm{mL}$ after $\mathrm{hCG}$ treatment was considered to be indicative of the presence of functioning testicular tissue and was defined as normal (16). Laboratory diagnosis of AIS was identified as normal-sized testes, absent Mullerian structures, normal follicle-stimulating hormone (FSH), normal/mildly elevated luteinizing hormone (LH) and normal/elevated baseline or hCG-stimulated $\mathrm{T}$ level, and normal T/DHT ratio (T-to-DHT ratio <20). A T/DHT ratio $\geq 20$ was accepted as suggestive of $5 \alpha-R D(17,18)$. Also for exclution of $46, \mathrm{XY}$ DSD causes related to congenital adrenal hyperplasia,cortisol, 17-hydroxyprogesterone (17-OHP), dehydroepiandrosterone sulfate (DHEA-S), and androstenedione (A) were also measured. A T-to-A ratio (T/A) $<0.8$ was accepted as suggestive of $17 \beta$-hydroxysteroid dehydrogenase $(17 \beta-H S D)$ deficiency $(11,12)$ and they were excluded.For statistical comparisons, the groups were divided into subgroups according to their genetic diagnosis and T/DHT ratios.

\section{Hormone Assays}

Adrenocorticotropic hormone, cortisol, DHEA-S, A and 17OHP were measured using the IMMULITE 2000 system (immunochemiluminescence assay; ICMA; Siemens AG, Berlin and Munich, Germany) while LH, FSH, and T were analyzed by electrochemiluminiscense immunoassay (Cobas, Roche Diagnostics, Mannheim, Germany). Enzymelinked immunosorbent assay kits were also used for the direct quantitative determination of DHT although different laboratory results using different reagents were included in the study as a result of retrospective design.

\section{Molecular Analysis}

Conventional karyotyping analysis were performed before molecular genetic investigations. Sequence variants of the SRD5A2 gene (NM_000348.3) screened in the cases who had T/DHT ratio $\geq 20$ whereas the $A R$ gene (NM_000044.4) was investigated by Sanger sequencing in patients who had the ratio $<20$ for pathogenic alterations. If no pathogenic alteration was detected in the first analysis, then the second genetic analysis was applied for SRD5A2 or AR, whichever was not analysed in the first run (Figure 1). Those who did not have a mutation in either AR or SRD5A2 constituted the undiagnosed group. Each group were further subgrouped according the T/DHT value. Pathogenic variants were confirmed by database search (Human Genome Mutation Database, ClinVar) and literature search and classified according to ACMG guideline $(19,20,21)$. Human splice finder analysis was used for variants with silent status (22). Segregation in family members were performed whenever available.

\section{Statistical Analysis}

The data were analyzed using Statistical Package for the Social Sciences (SPSS), version 21 (SPSS Inc., Chicago, IL, USA). Data were analyzed using descriptive statistical methods [mean, standard deviation, median, frequency, rate, ranges (minimum-maximum)] as well as some methods for comparing quantitative data. The results are given as median (minimum-maximum values) according to the distribution of data or as percentages, where appropriate. Mann-Whitney U test was used in the two-group comparisons of parameters without normal distribution. In comparison of three or more parameters without normal distribution Kruskal-Wallis test was used. A p value of less than 0.05 were considered statistically significant. 


\section{Results}

\section{Clinical Findings}

A total of 128 DSD patients from 125 unrelated families were enrolled in the study. The flowchart of the distribution of the patients is given in Figure 1.

On admission, the median age of AIS patients $(n=18)$ was 1.0 [minimum (min): 0.01, maximum (max): 17.4] years, in $5 \alpha-R D(n=14)$ it was 6.7 years (min: 0.01, max: 17.5), and 0.3 years (min: 0.01, max: 14.3) in the undiagnosed group $(n=96)(p<0.05)$. Review of patient charts indicated that Quigley scale grade and EMS on admission, reared gender, consanguinity, birth weight (BW) SDS and gestational age (GA, weeks) of patients were different between the three groups. BW SDS and GA were significantly higher in $5 \alpha-R D$ patients than the other two groups. Median (range) BW SDS in $5 \alpha-R D$ patients was 0.4 $(-1.5$ to 2.4$)$ whereas median BW SDS were $-1.0(-4.2$ to $2.0)$ in AIS and -1.5 (-7.7 to 3.3 ) in the undiagnosed group. Median values of GA in groups were also 40 (38-40) weeks in 5 $\alpha-\mathrm{RD}, 38$ (28-40) weeks in AIS and 38 (27-42) weeks in the undiagnosed group. SGA rate was significantly different between groups. None of the $5 \alpha-R D$ patients had SGA whereas the SGA rates in AIS and undiagnosed groups were $27.8 \%$ and $40.2 \%$, respectively. Consanguinity rate was higher in $5 \alpha$-RD patients than in both AIS and in the undiagnosed patients (Tables 1,2,3,4).

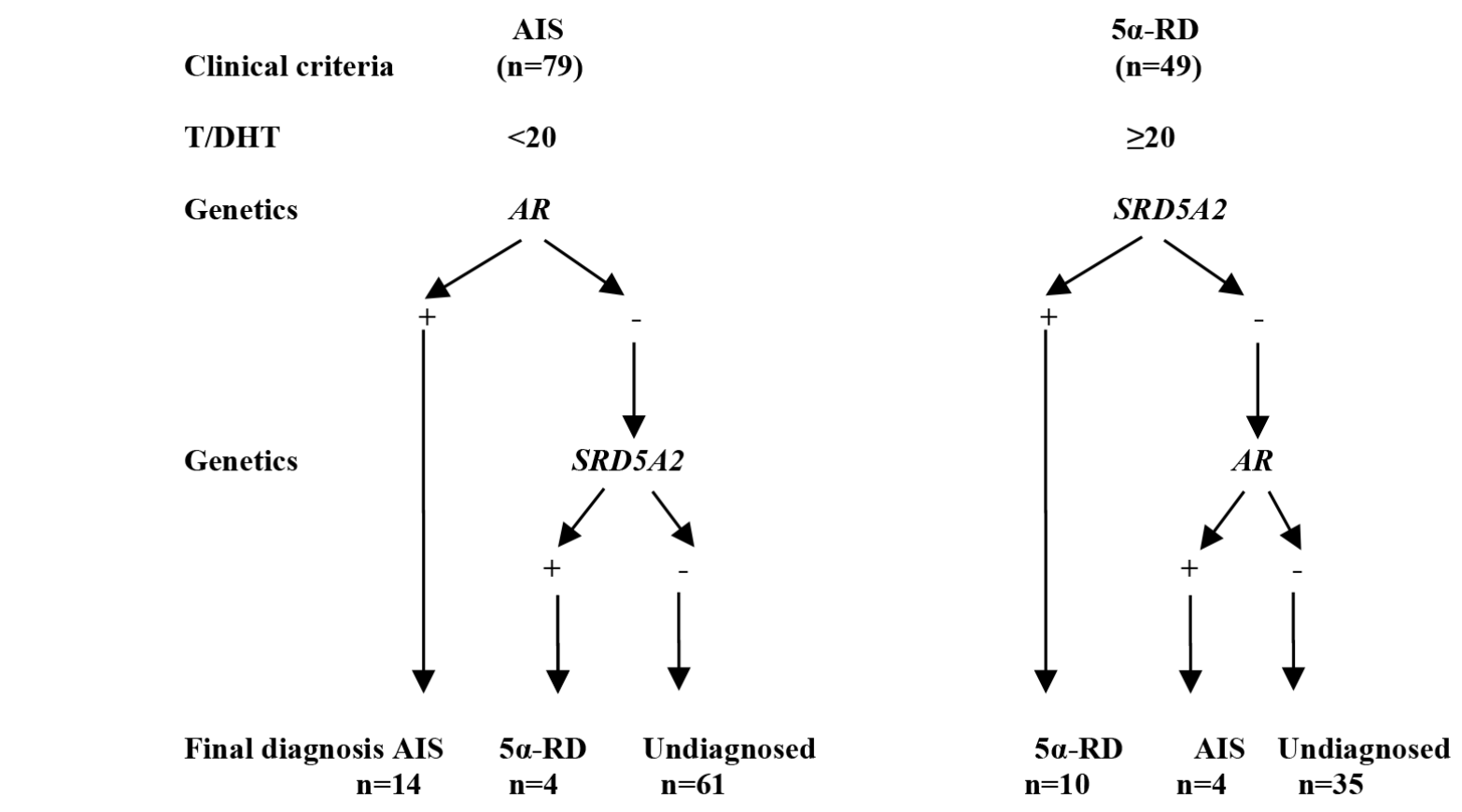

Comparisons of AIS and $5 \alpha-\mathrm{RD}$ groups with the undiagnosed group according to T/DHT ratios are detailed in Tables $1,2,3,4$, respectively. Although age at presentation did not differ between any subgroup of AIS and undiagnosed patients, $5 \alpha-R D$ patients presented later than undiagnosed patients (Tables 1,2,3,4). BWSDS and GA of undiagnosed patients were significantly lower than $5 \alpha$-RD patients, whereas it did not differ between the subgroups of AIS and undiagnosed patients (Tables 1,2,3,4). However, the percentages of SGA, AGA, and LGA were same between the subgroups. Symptoms on admission, additional findings, current anthropometry and pubertal status of all subgroups are also shown in Tables 1,2,3,4. Additional findings or diagnoses, including SGA or prematurity, were significantly lower in $5 \alpha-R D$ subgroups than in the undiagnosed subgroups whereas it was not different between AIS and undiagnosed subgroups. However, other disease comorbidities (kidney disease, congenital heart disease, anal atresia and autism) were detected only in the undiagnosed group (Tables 1,2,3,4). According to Quigley scale, CAIS grade rate was higher in all subgroups of both AIS and $5 \alpha$-RD patients than in the undiagnosed subgroups (Tables 1,2,3,4). Comparison of EMS within subgroups differed significantly except between genetically proven AIS and undiagnosed patients with a normal T/DHT value $(<20)$ (Tables 1,2,3,4). Reared gender was also different between the subgroups (Tables $1,2,3,4$ ).

Figure 1. Analysis of the patients with 46,XY DSD

$5 \alpha-R D$ : $5 \alpha$-reductase deficiency 


\begin{tabular}{|c|c|c|c|}
\hline & Mutation $(+)$ & Mutation (-) & $\mathrm{p}$ \\
\hline T/DHT: Normal & $\mathrm{n}=14$ & $n=61$ & \\
\hline Age at presentation (years) & $1.8 \pm 3.1$ & $1.3 \pm 2.0$ & 0.98 \\
\hline Consanguinity, n (\%) & $25 \%(n=3)$ & $23 \%(n=14)$ & 0.87 \\
\hline Birth weight SDS & $-0.8 \pm 1.9$ & $-1.7 \pm 2.0$ & 0.19 \\
\hline Gestational age (weeks) & $37 \pm 4.1$ & $36.6 \pm 3.7$ & 0.46 \\
\hline Gestational age groups (weeks), n (\%) & & & 0.4 \\
\hline$<37$ & $21.4 \%(n=3)$ & $39.3 \%(n=24)$ & \\
\hline$\geq 37$ & $78.5 \%(n=11)$ & $60.6 \%(n=37)$ & \\
\hline BW groups, n (\%) & & & 0.6 \\
\hline AGA $(-2$ to +2 SDS), $n(\%)$ & $64.3 \%(n=9)$ & $54 \%(n=33)$ & \\
\hline SGA $(<-2$ SDS), n $(\%)$ & $28.6 \%(n=4)$ & $42.6 \%(n=26)$ & \\
\hline $\operatorname{LGA}(>+2 \mathrm{SDS}), \mathrm{n}(\%)$ & $7.1 \%(n=1)$ & $3.3 \%(n=2)$ & \\
\hline Symptom on admission & & & 0.07 \\
\hline Ambiguous genitalia & $78.5 \%(n=11)$ & $93.4 \%(n=57)$ & \\
\hline Same diagnosis siblings & - & $1.6 \%(n=1)$ & \\
\hline Mass in groin & $14.2 \%(n=2)$ & $1.6 \%(n=1)$ & \\
\hline Undescended testes & - & $1.6 \%(n=1)$ & \\
\hline Micropenis & - & $1.6 \%(n=1)$ & \\
\hline Finding testis during inguinal hernia surgery & $7.1 \%(n=1)$ & - & \\
\hline \multicolumn{4}{|l|}{ At current } \\
\hline Age (years) & $6.7 \pm 0.9$ & $6.5 \pm 5.4$ & 0.40 \\
\hline Weight SDS & $-0.4 \pm 1.6$ & $-0.7 \pm 1.2$ & 0.69 \\
\hline Height SDS & $-0.3 \pm 1.4$ & $-1.0 \pm 1.1$ & 0.16 \\
\hline Puberty status & & & 0.21 \\
\hline Tanner stage 1 & $78.5 \%(n=11)$ & $77 \%(n=47)$ & \\
\hline Tanner stage 2 & $7.1 \%(n=1)$ & $8.2 \%(n=5)$ & \\
\hline Tanner stage 3 & $7.1 \%(n=1)$ & $1.6(n=1)$ & \\
\hline Tanner stage 4 & $7.1 \%(n=1)$ & - & \\
\hline Tanner stage 5 & - & $9.8 \%(n=6)$ & \\
\hline Mini puberty & - & $1.6 \%(n=1)$ & \\
\hline Estrogen replacement after gonadectomy & - & $1.6 \%(n=1)$ & \\
\hline Reared gender, n (\%) & & & $<0.01 *$ \\
\hline Male & $71.4 \%(n=10)$ & $96.7 \%(n=59)$ & \\
\hline Female & $28.5 \%(n=4)$ & $1.6 \%(n=1)$ & \\
\hline Female reared, changed identity after diagnosis & - & $1.6 \%(n=1)$ & \\
\hline Ouigley scale & & & 0.06 \\
\hline 2 & $7.1 \%(n=1)$ & $18 \%(n=11)$ & \\
\hline 3 & $35.7 \%(n=5)$ & $57.4 \%(n=35)$ & \\
\hline 4 & $14.2 \%(n=2)$ & $11.5 \%(n=7)$ & \\
\hline 5 & $7.1 \%(n=1)$ & $4.9 \%(n=3)$ & \\
\hline $6 / 7$ & $28.5 \%(n=4)$ & $1.6 \%(n=1)$ & \\
\hline Applied after operation & $7.1 \%(n=1)$ & $6.5 \%(n=4)$ & \\
\hline EMS & & & 0.23 \\
\hline 1 & $5.5 \%(n=1)$ & $1.6 \%(n=1)$ & \\
\hline 2 & $16.7 \%(n=3)$ & $0 \%(n=0)$ & \\
\hline 4 & $11.1 \%(n=2)$ & $16.4 \%(n=10)$ & \\
\hline 5 & $0 \%(n=0)$ & $23 \%(n=14)$ & \\
\hline 6 & $22.2 \%(n=4)$ & $19.7 \%(n=12)$ & \\
\hline 7 & $11.1 \%(n=2)$ & $27.9 \%(n=17)$ & \\
\hline 8 & $5.5 \%(n=1)$ & $4.9 \%(n=3)$ & \\
\hline Applied after operation & $5.5 \%(n=1)$ & $6.5 \%(n=4)$ & \\
\hline
\end{tabular}




\begin{tabular}{|c|c|c|c|}
\hline & Mutation $(+)$ & Mutation (-) & $\mathrm{p}$ \\
\hline AIS groups, n (\%) & & & $0.02 *$ \\
\hline PAIS & $71.4 \%(n=10)$ & $98.4 \%(n=60)$ & \\
\hline CAIS & $28.5 \%(n=4)$ & $1.6 \%(n=1)$ & \\
\hline Additional findings & & & 0.19 \\
\hline None & $57.1 \%(n=8)$ & $36 \%(n=22)$ & \\
\hline Prematurity & $21.4 \%(n=3)$ & $34.4 \%(n=21)$ & \\
\hline IUGR & - & $9.8 \%(n=6)$ & \\
\hline Multicystic dysplastic kidney & - & $3.3 \%(n=2)$ & \\
\hline Congenital heart disease & - & $4.9 \%(n=3)$ & \\
\hline Anal atresia & - & $3.3 \%(n=2)$ & \\
\hline Nephrolithiasis & - & $1.6 \%(n=1)$ & \\
\hline Autism & - & $1.6 \%(n=1)$ & \\
\hline Gonadoblastoma & - & $1.6 \%(n=1)$ & \\
\hline Infant of diabetic mother & - & $1.6 \%(n=1)$ & \\
\hline Klinefelter syndrome & $7.1 \%(n=1)$ & $1.6 \%(n=1)$ & \\
\hline $47, X Y Y$ & $7.1 \%(\mathrm{n}=1)$ & - & \\
\hline Agenesis of the corpus callosum & $7.1 \%(n=1)$ & - & \\
\hline Target height SDS & $-0.8 \pm 0.2$ & $-0.9 \pm 0.09$ & 0.97 \\
\hline \multicolumn{4}{|c|}{ Laboratory results according to the age } \\
\hline $0-6$ months & $\mathrm{n}=7$ & $n=26$ & \\
\hline Age (months) & $2.1 \pm 1.5$ & $1.6 \pm 1.6$ & 0.38 \\
\hline LH (mIU/mL) & $4.9 \pm 4.3$ & $4.1 \pm 3.7$ & 0.62 \\
\hline FSH $(\mathrm{mIU} / \mathrm{mL})$ & $1.7 \pm 1.2$ & $2.2 \pm 1.6$ & 0.42 \\
\hline Basal T (ng/mL) & $1.5 \pm 1.4$ & $1.6 \pm 0.8$ & 0.76 \\
\hline Basal DHT (ng/mL) & $0.3 \pm 0.2$ & $0.4 \pm 0.1$ & 0.59 \\
\hline Basal T/DHT ratio & $10.5 \pm 7.3$ & $6.9 \pm 6.3$ & 0.22 \\
\hline Prepubertal & $\mathrm{n}=7$ & $\mathrm{n}=38$ & \\
\hline Age (years) & $4.0 \pm 2.9$ & $3.8 \pm 2.9$ & 0.67 \\
\hline $\mathrm{LH}(\mathrm{mIU} / \mathrm{mL})$ & $0.7 \pm 0.9$ & $0.5 \pm 1.6$ & 0.23 \\
\hline FSH (mIU/mL) & $1.2 \pm 1.0$ & $1.5 \pm 2.5$ & 0.86 \\
\hline Basal T (ng/mL) & $0.3 \pm 0.4$ & $0.2 \pm 0.4$ & 0.82 \\
\hline Stimulated T (ng/mL) & $5.1 \pm 2.9$ & $4.3 \pm 2.4$ & 0.38 \\
\hline Stimulated DHT (ng/mL) & $1.5 \pm 1.6$ & $0.6 \pm 0.5$ & 0.12 \\
\hline Stimulated T/DHT & $4.7 \pm 2.8$ & $9.3 \pm 1.3$ & $0.04 *$ \\
\hline Pubertal & $n=3$ & $\mathrm{n}=8$ & \\
\hline Age (years) & $10.8 \pm 0.3$ & $12.7 \pm 3.0$ & 0.13 \\
\hline $\mathrm{LH}(\mathrm{mIU} / \mathrm{mL})$ & $2.4 \pm 4.2$ & $3.8 \pm 5.0$ & 0.69 \\
\hline FSH (mIU/mL) & $2 \pm 4.9$ & $6.5 \pm 9.4$ & 0.56 \\
\hline Basal T (ng/mL) & $4.3 \pm 3.1$ & $2.6 \pm 2.0$ & 0.51 \\
\hline Basal DHT (ng/mL) & $0.5 \pm 0.2$ & $0.4 \pm 0.3$ & 0.91 \\
\hline Basal T/DHT & $9.5 \pm 4.5$ & $5.8 \pm 3.0$ & 0.16 \\
\hline
\end{tabular}

\section{Endocrine Data}

Laboratory results of AIS, $5 \alpha-R D$ and the undiagnosed groups according to their T/DHT ratios are detailed in Tables 1, 2, 3 and $4.22 .2 \%(n=4)$ of AIS patients and $36.5 \%(n=35)$ of undiagnosed patients had T/DHT ratio $\geq 20$, whereas $28.6 \%(n=4)$ of $5 \alpha-R D$ patients had T/DHT ratio $<20$
(Table 1-4). T/DHT ratios were obtained in $57 \%(\mathrm{n}=73)$ during hCG test, whereas the remaining were obtained by basal hormone levels during puberty or minipuberty. Twenty four patients had more than one T/DHT ratio according to their ages. The mean values of T/DHT ratios used for hormonal diagnosis were significantly different between 
Table 2. Comparison of clinical and hormonal features of genetically proven AIS and undiagnosed patients with a high T/DHT value $(\geq 20)$

\begin{tabular}{|c|c|c|c|}
\hline & Mutation $(+)$ & Mutation (-) & $\mathrm{p}$ \\
\hline T/DHT: High & $n=4$ & $\mathrm{n}=35$ & \\
\hline Age at presentation (years) & $4.0 \pm 4.4$ & $1.3 \pm 2.8$ & 0.11 \\
\hline Consanguinity, n (\%) & $0 \%(n=0)$ & $25.8 \%(n=8)$ & 0.38 \\
\hline Birth weight SDS & $-0.7 \pm 1.9$ & $-1.2 \pm 1.6$ & 0.7 \\
\hline Gestational age (weeks) & $35.0 \pm 7.0$ & $35.9 \pm 4.1$ & 0.93 \\
\hline Gestational age groups (weeks), n (\%) & & & 0.84 \\
\hline$<37$ & $25 \%(n=1)$ & $40 \%(n=14)$ & \\
\hline$\geq 37$ & $75 \%(n=3)$ & $60 \%(n=21)$ & \\
\hline BW groups, n (\%) & & & 0.88 \\
\hline AGA $(-2$ to +2 SDS), $\mathrm{n}(\%)$ & $75 \%(n=3)$ & $60 \%(n=21)$ & \\
\hline $\mathrm{SGA}(<-2 \mathrm{SDS}), \mathrm{n}(\%)$ & $25 \%(n=1)$ & $34.3 \%(n=12)$ & \\
\hline LGA ( > + 2 SDS), n (\%) & $0 \%(n=0)$ & $5.7 \%(n=2)$ & \\
\hline Symptom on admission & & & $<0.01^{*}$ \\
\hline Ambiguous genitalia & $75 \%(n=3)$ & $94.3 \%(n=33)$ & \\
\hline Mass in groin & - & - & \\
\hline Undescended testes & - & $2.8 \%(n=1)$ & \\
\hline Micropenis & - & $2.8 \%(n=1)$ & \\
\hline Finding testis during inguinal hernia surgery & $25 \%(n=1)$ & - & \\
\hline \multicolumn{4}{|l|}{ At current } \\
\hline Age (years) & $7.7 \pm 8.6$ & $3.9 \pm 4.7$ & 0.34 \\
\hline Weight SDS & $0.6 \pm 1.8$ & $-0.7 \pm 1.2$ & 0.20 \\
\hline Height SDS & $-0.3 \pm 0.6$ & $-0.9 \pm 1.3$ & 0.38 \\
\hline Puberty status & & & 0.07 \\
\hline Tanner stage 1 & $25 \%(n=1)$ & $82.9 \%(n=29)$ & \\
\hline Tanner stage 2 & - & - & \\
\hline Tanner stage 3 & - & - & \\
\hline Tanner stage 4 & - & - & \\
\hline Tanner stage 5 & - & $11.4 \%(n=4)$ & \\
\hline Mini puberty & - & $5.7 \%(n=2)$ & \\
\hline Estrogen replacement after gonadectomy & $75 \%(n=3)$ & - & \\
\hline Reared gender, n (\%) & & & $0.04^{*}$ \\
\hline Male & $25 \%(n=1)$ & $94.3 \%(\mathrm{n}=33)$ & \\
\hline Female & $75 \%(n=3)$ & $2.8 \%(n=1)$ & \\
\hline Female reared, changed identity after diagnosis & - & $2.8 \%(n=1)$ & \\
\hline Ouigley scale & & & $<0.01^{*}$ \\
\hline 2 & - & $8.6 \%(n=3)$ & \\
\hline 3 & $25 \%(n=1)$ & $85.7 \%(n=30)$ & \\
\hline 4 & - & $2.8 \%(n=1)$ & \\
\hline 5 & - & $2.8 \%(n=1)$ & \\
\hline $6 / 7$ & $75 \%(n=3)$ & - & \\
\hline EMS & & & 0.01 * \\
\hline 1 & $50 \%(n=2)$ & $0 \%(n=0)$ & \\
\hline 2 & $25 \%(n=1)$ & $0 \%(n=0)$ & \\
\hline 3 & $0 \%(n=0)$ & $2.9 \%(n=1)$ & \\
\hline 4 & $0 \%(n=0)$ & $17.1 \%(n=6)$ & \\
\hline 5 & $0 \%(n=0)$ & $8.6 \%(n=3)$ & \\
\hline 6 & $25 \%(n=1)$ & $22.9 \%(n=8)$ & \\
\hline 7 & $0 \%(n=0)$ & $40 \%(n=14)$ & \\
\hline 8 & $0 \%(n=0)$ & $8.6 \%(n=3)$ & \\
\hline \multicolumn{4}{|l|}{ AIS groups, n (\%) } \\
\hline PAIS & $25 \%(n=1)$ & $100 \%(n=35)$ & $<0.01 *$ \\
\hline CAIS & $75 \%(\mathrm{n}=3)$ & - & \\
\hline
\end{tabular}




\begin{tabular}{|c|c|c|c|}
\hline & Mutation (+) & Mutation (-) & $\mathrm{p}$ \\
\hline Additional findings & & & 0.95 \\
\hline None & $75 \%(n=3)$ & $51.4 \%(n=18)$ & \\
\hline Prematurity & $25 \%(\mathrm{n}=1)$ & $34.3 \%(n=12)$ & \\
\hline IUGR & - & $11.4 \%(n=4)$ & \\
\hline Anal atresia & - & $2.8 \%(n=1)$ & \\
\hline Target height SDS & $-0.8 \pm 0.3$ & $-0.8 \pm 0.1$ & 0.93 \\
\hline \multicolumn{4}{|c|}{ Laboratory results according to the age } \\
\hline $0-6$ months & $n=0$ & $n=20$ & \\
\hline Age (months) & - & $2.5 \pm 2.1$ & - \\
\hline LH (mIU/mL) & - & $3.9 \pm 3.4$ & - \\
\hline FSH (mIU/mL) & - & $1.8 \pm 1.4$ & - \\
\hline Basal T (ng/mL) & - & $1.8 \pm 1.5$ & - \\
\hline Basal DHT (ng/mL) & - & $0.08 \pm 0.05$ & - \\
\hline Basal T/DHT & - & $40.0 \pm 18.5$ & - \\
\hline Prepubertal & $\mathrm{n}=2$ & $\mathrm{n}=19$ & \\
\hline Age (years) & $5.2 \pm 5.0$ & $2.2 \pm 2.2$ & 0.23 \\
\hline LH (mIU/mL) & $0.3 \pm 0.1$ & $0.3 \pm 0.2$ & 0.76 \\
\hline FSH (mIU/mL) & $1.8 \pm 1.6$ & $0.8 \pm 0.5$ & 0.23 \\
\hline Basal T (ng/mL) & $0.05 \pm 0.07$ & $0.05 \pm 0.07$ & 0.12 \\
\hline Stimulated T (ng/mL) & $4.6 \pm 2.2$ & $4.0 \pm 2.9$ & 0.95 \\
\hline Stimulated DHT (ng/mL) & $0.05 \pm 0.07$ & $0.2 \pm 0.4$ & 0.58 \\
\hline Stimulated T/DHT & $94.8 \pm 28.7$ & $81.6 \pm 15.9$ & 0.49 \\
\hline Pubertal & $n=3$ & $n=3$ & \\
\hline Age (years) & $12.3 \pm 2.7$ & $12.3 \pm 2.7$ & 0.22 \\
\hline LH (mIU/mL) & $23.9 \pm 3.0$ & $6.1 \pm 2.9$ & $0.04 *$ \\
\hline FSH (mIU/mL) & $20.6 \pm 10.4$ & $6.6 \pm 5.7$ & 0.12 \\
\hline Basal T (ng/mL) & $6.5 \pm 1.9$ & $4.4 \pm 2.2$ & 0.28 \\
\hline Basal DHT (ng/mL) & $0.2 \pm 0.08$ & $0.09 \pm 0.1$ & 0.64 \\
\hline Basal T/DHT ratio & $55.8 \pm 29.7$ & $57.3 \pm 21.2$ & 0.31 \\
\hline
\end{tabular}

AIS, $5 \alpha-R D$ and undiagnosed groups $(p<0.05)$. 5 $\alpha-R D$ patients had significant higher T/DHT ratios than both the AIS and undiagnosed groups. Although basal T/DHT ratio during minipuberty or puberty did not differ, stimulated T/ DHT ratio in the prepubertal period was significantly lower in the AIS subgroup with T/DHT $<20$ than in the undiagnosed subgroup with $\mathrm{T} / \mathrm{DHT}<20$ (Table 1). In contrast, the stimulated T/DHT ratio in the prepubertal period was significantly higher in the $5 \alpha$-RD subgroup with T/DHT $<20$ than in the undiagnosed subgroup with T/DHT $<20$ (Table 3). Clinical and laboratory findings of genetically diagnosed AIS and $5 \alpha-R D$ patients arepresented in Supplementary Table 1 and 2. Only pubertal LH was significantly higher in AIS patients than the undiagnosed group when T/DHT $\geq 20$.

\section{Molecular Genetics}

Cytogeneticaly, 32 patients had definitive diagnosis and four patients had non-46,XY karyotypes (three with 47,XXY, and one with $47, \mathrm{XYY}$ ). Fourteen patients were investigated only for AR, 10 only for SRD5A2 and 104 patients were investigated for both genes. Variants attributed to disease were found in 32 (24\%) patients, whereas the others $(75 \%, n=96)$ remained undiagnosed for molecular genetic base. In the total cohort, $14 \%$ of patients $(n=18)$ were molecularly diagnosed as AIS, and $10.9 \%(n=14)$ had $5 \alpha-R D$ genetically. Molecular genetic test results showed four novel $A R$ variants (c.94G > T, p.Glu32*; c.330G > C, p.Leu110 = ; c.2084C > T, p.Pro695Leu; c.2585_2592delAGCTCCTG, p.(Lys862Argfs*16). Among those, c.94G > T and c.2585_2592delAGCTCCTG were classified as pathogenic, c.2084C $>\mathrm{T}$ as likely pathogenic, while silent change (c.330G > C) as likely benign. In in silico analysis for human splicing finder, c.330G > C is not expected to have significant impact on splicing signals (22). This silent change would cause the alteration of leucine encoded by CTG to CTC. In a study of translation-selection model of human genome, it was shown that CTG is the major codon for leucine-tRNA, being more abundant in a translation environment, an important 


\begin{tabular}{|c|c|c|c|}
\hline & Mutation $(+)$ & Mutation (-) & $\mathrm{p}$ \\
\hline T/DHT: Normal & $\mathrm{n}=4$ & $n=61$ & \\
\hline Age at presentation (years) & $12.0 \pm 4.9$ & $1.3 \pm 2.0$ & $<0.01 *$ \\
\hline Consanguinity, n (\%) & $75 \%(n=3)$ & $23 \%(n=14)$ & $0.02 *$ \\
\hline Birth weight SDS & $0.0 \pm 1.0$ & $-1.7 \pm 2.0$ & 0.07 \\
\hline Gestational age (weeks) & $39.7 \pm 0.5$ & $36.6 \pm 3.7$ & $0.04 *$ \\
\hline Gestational age groups (weeks), n (\%) & & & 0.12 \\
\hline$<37$ & $0 \%(n=0)$ & $39.3 \%(n=24)$ & \\
\hline$\geq 37$ & $100 \%(n=4)$ & $60.6 \%(n=37)$ & \\
\hline BW groups, n (\%) & & & 0.19 \\
\hline AGA $(-2$ to +2 SDS), n (\%) & $100 \%(n=4)$ & $54 \%(n=33)$ & \\
\hline SGA (<-2 SDS), n (\%) & $0 \%(n=0)$ & $42.6 \%(n=26)$ & \\
\hline LGA ( > + 2 SDS), n (\%) & $0 \%(n=0)$ & $3.3 \%(n=2)$ & \\
\hline Symptom on admission & & & $<0.01 *$ \\
\hline Ambiguous genitalia & 0 & $93.4 \%(n=57)$ & \\
\hline Same diagnosis siblings & $50 \%(n=2)$ & $1.6 \%(n=1)$ & \\
\hline Mass in groin & $50 \%(n=2)$ & $1.6 \%(n=1)$ & \\
\hline Undescended testes & 0 & $1.6 \%(n=1)$ & \\
\hline Micropenis & 0 & $1.6 \%(n=1)$ & \\
\hline \multicolumn{4}{|l|}{ At current evaluation } \\
\hline Age (years) & $15.9 \pm 2.8$ & $6.5 \pm 5.4$ & $<0.01 *$ \\
\hline Weight SDS & $-0.8 \pm 0.7$ & $-0.7 \pm 1.2$ & 0.82 \\
\hline Height SDS & $-0.6 \pm 0.6$ & $-1.0 \pm 1.1$ & 0.56 \\
\hline Puberty status & & & $<0.01^{*}$ \\
\hline Tanner Stage 1 & - & $77 \%(\mathrm{n}=47)$ & \\
\hline Tanner Stage 2 & $25 \%(n=1)$ & $8.2 \%(n=5)$ & \\
\hline Tanner Stage 3 & - & $1.6(n=1)$ & \\
\hline Tanner Stage 4 & - & - & \\
\hline Tanner Stage 5 & - & $9.8 \%(n=6)$ & \\
\hline Mini puberty & - & $1.6 \%(n=1)$ & \\
\hline Estrogen replacement after gonadectomy & $75 \%(n=3)$ & $1.6 \%(n=1)$ & \\
\hline Reared gender, n (\%) & & & $<0.01 *$ \\
\hline Male & - & $96.7 \%(n=59)$ & \\
\hline Female & $75 \%(n=3)$ & $1.6 \%(n=1)$ & \\
\hline Female reared, changed identity after diagnosis & $25 \%(n=1)$ & $1.6 \%(n=1)$ & \\
\hline Ouigley scale on admission & & & $<0.01 *$ \\
\hline 2 & - & $18 \%(n=11)$ & \\
\hline 3 & $25 \%(n=1)$ & $57.4 \%(n=35)$ & \\
\hline 4 & - & $11.5 \%(\mathrm{n}=7)$ & \\
\hline 5 & $50 \%(n=2)$ & $4.9 \%(n=3)$ & \\
\hline $6 / 7$ & $25 \%(n=1)$ & $1.6 \%(n=1)$ & \\
\hline Applied after operation & - & $6.5 \%(n=4)$ & \\
\hline EMS score & & & $<0.01 *$ \\
\hline 1 & $0 \%(n=0)$ & $1.6 \%(n=1)$ & \\
\hline 2 & $25 \%(n=1)$ & $0 \%(n=0)$ & \\
\hline 4 & $0 \%(n=0)$ & $16.4 \%(n=10)$ & \\
\hline 5 & $0 \%(n=0)$ & $23 \%(n=14)$ & \\
\hline 6 & $0 \%(n=0)$ & $19.7 \%(n=12)$ & \\
\hline 7 & $0 \%(n=0)$ & $27.9 \%(n=17)$ & \\
\hline 8 & $75 \%(n=3)$ & $4.9 \%(n=3)$ & \\
\hline Applied after operation & & $6.5 \%(n=4)$ & \\
\hline
\end{tabular}




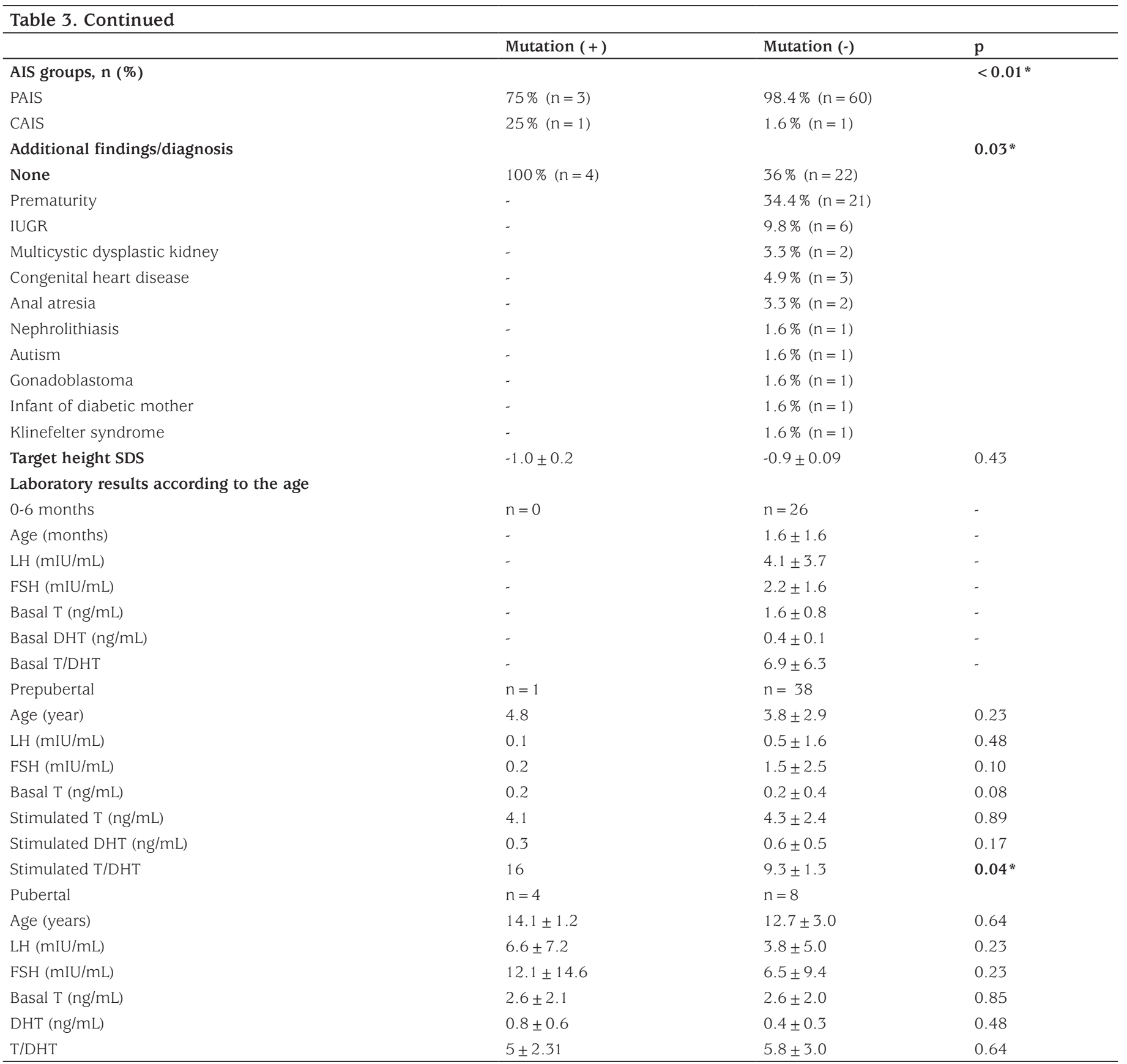

AGA: appropriate for gestational age, AIS: androgenin sensitivity syndrome, BW: birth weight, CAIS: complete androgen insensitivity syndrome, DHT: dihydrotestosterone, EMS: external masculinization score, FSH: follicle-stimulating hormone, IUGR: intrauterine growth retardation, LGA: large for gestational age, LH: luteinizing hormone, PAIS: partial androgen insensitivity syndrome, SGA: small for gestational age, T: testosterone, SDS: standard deviation score

factor determining translational efficiency (23). Presently, there is no sufficient evidence to support a causative status of C.330G > C and segregation analysis for this family was not performed. The most frequent pathogenic $A R$ variant in the study was c.1174C > T, (p.Pro392Ser) with a frequency of $33.3 \%(n=6)$ in all AIS patients (Table 5). All of the patients with this mutation presented with PAIS clinically. All of patients with CAIS ( $n=7,38.9 \%$ ) had different mutations (two of them novel). However two siblings with c.2676T > A (p.Phe892Leu) mutation had different clinical presentations (one PAIS, one CAIS) and one of these siblings with PAIS also had a 47,XXY karyotype (Supplementary Table 1). The most frequent SRD5A2 mutations were c.164T > A (p.Leu55Gln), c.453delC (p.(Leu152Tyrfs*8) and c.193G > C (p.Ala65Pro). Two patients with c.453delC (p.(Leu152Tyrfs*8)) also had different Quigley scores, assigend as PAIS in one and CAIS in the other. A patient who had heterozygous mutations with c.164T > A, p.Leu55Gln and c.269A > C (p.His90Pro) presented with a CAIS phenotype (Supplementary Table 2). 


\begin{tabular}{|c|c|c|c|}
\hline & Mutation $(+)$ & Mutation (-) & $\mathrm{p}$ \\
\hline T/DHT: High & $\mathrm{n}=10$ & $\mathrm{n}=35$ & \\
\hline Age at presentation (years) & $6.6 \pm 7.2$ & $1.3 \pm 2.8$ & $0.04^{*}$ \\
\hline Birth weight SDS & $0.6 \pm 1.4$ & $-1.2 \pm 1.6$ & $0.007 *$ \\
\hline Gestational age (weeks) & $39.2 \pm 0.8$ & $35.9 \pm 4.1$ & $0.02^{*}$ \\
\hline Gestational age (weeks), n (\%) & & & $0.03 *$ \\
\hline$<37$ & $0 \%(n=0)$ & $40 \%(n=14)$ & \\
\hline$\geq 37$ & $100 \%(n=10)$ & $60 \%(n=21)$ & \\
\hline Consanguinity, n (\%) & $50 \%(\mathrm{n}=5)$ & $25.8 \%(\mathrm{n}=8)$ & 0.14 \\
\hline BW groups, n (\%) & & & 0.10 \\
\hline AGA (-2 to +2 SDS), n (\%) & $90 \%(n=9)$ & $60 \%(n=21)$ & \\
\hline $\mathrm{SGA}(<-2 \mathrm{SDS}), \mathrm{n}(\%)$ & $0 \%(n=0)$ & $34.3 \%(n=12)$ & \\
\hline LGA ( > + 2 SDS) n (\%) & $10 \%(n=1)$ & $5.7 \%(n=2)$ & \\
\hline Symptom on admission & & & 0.07 \\
\hline Ambiguous genitalia & $60 \%(n=6)$ & $94.3 \%(n=33)$ & \\
\hline Mass in groin & $10 \%(n=1)$ & - & \\
\hline Undescended testes & - & $2.8 \%(n=1)$ & \\
\hline Micropenis & - & $2.8 \%(n=1)$ & \\
\hline Primary amenorrhea & $10 \%(\mathrm{n}=1)$ & - & \\
\hline Virilization in puberty & $10 \%(n=1)$ & - & \\
\hline Cliteromegali & $10 \%(n=1)$ & - & \\
\hline \multicolumn{4}{|l|}{ At current evaluation } \\
\hline Age (years) & $6.9 \pm 6.0$ & $3.9 \pm 4.7$ & $0.03 *$ \\
\hline Weight SDS & $0.6 \pm 1.0$ & $-0.7 \pm 1.2$ & $0.01 *$ \\
\hline Height SDS & $0.3 \pm 0.9$ & $-0.9 \pm 1.3$ & $0.02^{*}$ \\
\hline Puberty status & & & 0.95 \\
\hline Tanner stage 1 & $60 \%(n=6)$ & $82.9 \%(n=29)$ & \\
\hline Tanner stage 2 & - & - & \\
\hline Tanner stage 3 & $10 \%(n=1)$ & - & \\
\hline Tanner stage 4 & $10 \%(n=1)$ & - & \\
\hline Tanner stage 5 & $10 \%(n=1)$ & $11.4 \%(n=4)$ & \\
\hline Mini puberty & - & $5.7 \%(n=2)$ & \\
\hline Estrogen replacement after gonadectomy & $10 \%(n=1)$ & - & \\
\hline Reared gender, n (\%) & & & $0.02 *$ \\
\hline Male & $50 \%(n=5)$ & $94.3 \%(\mathrm{n}=33)$ & \\
\hline Female & $10 \%(n=1)$ & $2.8 \%(n=1)$ & \\
\hline Female reared, changed identity after diagnosis & $4 \%(n=4)$ & $2.8 \%(\mathrm{n}=1)$ & \\
\hline Ouigley scale & & & $0.03^{*}$ \\
\hline 2 & - & $8.6 \%(n=3)$ & \\
\hline 3 & $50 \%(n=5)$ & $85.7 \%(n=30)$ & \\
\hline 4 & $10 \%(n=1)$ & $2.8 \%(n=1)$ & \\
\hline 5 & $20 \%(n=2)$ & $2.8 \%(n=1)$ & \\
\hline $6 / 7$ & $20 \%(n=2)$ & - & \\
\hline EMS score & & & $<0.01^{*}$ \\
\hline 1 & $10 \%(n=1)$ & $0 \%(n=0)$ & \\
\hline 2 & $10 \%(n=1)$ & $0 \%(n=0)$ & \\
\hline 3 & $\%(n=0)$ & $2.9 \%(n=1)$ & \\
\hline 4 & $30 \%(n=3)$ & $17.1 \%(n=6)$ & \\
\hline 5 & $10 \%(n=1)$ & $8.6 \%(n=3)$ & \\
\hline 6 & $10 \%(n=1)$ & $22.9 \%(n=8)$ & \\
\hline 7 & $\%(n=0)$ & $40 \%(n=14)$ & \\
\hline 8 & $30 \%(n=3)$ & $8.6 \%(n=3)$ & \\
\hline
\end{tabular}




\begin{tabular}{|c|c|c|c|}
\hline & Mutation $(+)$ & Mutation (-) & $\mathrm{p}$ \\
\hline AIS groups, $\mathrm{n}(\%)$ & & & 0.03 * \\
\hline PAIS & $80 \%(n=8)$ & $100 \%(n=35)$ & \\
\hline CAIS & $20 \%(n=2)$ & - & \\
\hline Additional findings/diagnosis & & & $0.04^{*}$ \\
\hline None & $90 \%(n=9)$ & $51.4 \%(n=18)$ & \\
\hline Prematurity & - & $34.3 \%(\mathrm{n}=12)$ & \\
\hline IUGR & - & $11.4 \%(n=4)$ & \\
\hline Klinefelter syndrome & $10 \%(\mathrm{n}=1)$ & $2.8 \%(n=1)$ & \\
\hline Anal atresia & - & $51.4 \%(n=18)$ & \\
\hline Target height SDS & $-0.9 \pm 0.1$ & $-0.8 \pm 0.1$ & 0.35 \\
\hline \multicolumn{4}{|c|}{ Laboratory results according to the age } \\
\hline $0-6$ months & $\mathrm{n}=2$ & $\mathrm{n}=20$ & \\
\hline Age (months) & $1.5 \pm 2.2$ & $2.5 \pm 2.1$ & 0.33 \\
\hline LH (mIU/mL) & $4.7 \pm 1.9$ & $3.9 \pm 3.4$ & 0.76 \\
\hline FSH (mIU/mL) & $2.3 \pm 1.0$ & $1.8 \pm 1.4$ & 0.31 \\
\hline Basal T (ng/mL) & $2.1 \pm 2.0$ & $1.8 \pm 1.5$ & 0.73 \\
\hline Basal DHT (ng/mL) & $0.5 \pm 0.02$ & $0.08 \pm 0.05$ & 0.13 \\
\hline Basal T/DHT & $52.7 \pm 19.7$ & $40.0 \pm 18.5$ & 0.13 \\
\hline Prepubertal & $\mathrm{n}=6$ & $\mathrm{n}=19$ & \\
\hline Age (years) & $4.3 \pm 2.3$ & $2.2 \pm 2.2$ & 0.01 * \\
\hline LH (mIU/mL) & $0.4 \pm 0.4$ & $0.3 \pm 0.2$ & 0.89 \\
\hline FSH (mIU/mL) & $0.9 \pm 0.5$ & $0.8 \pm 0.5$ & 0.47 \\
\hline Basal T (ng/mL) & $0.07 \pm 0.07$ & $0.05 \pm 0.07$ & 0.43 \\
\hline Stimulated T (ng/mL) & $3.4 \pm 2.4$ & $4.0 \pm 2.9$ & 0.52 \\
\hline Stimulated DHT (ng/mL) & $0.08 \pm 0.07$ & $0.2 \pm 0.4$ & 0.64 \\
\hline Stimulated T/DHT & $67.4 \pm 27.5$ & $81.6 \pm 15.9$ & 0.50 \\
\hline Pubertal & $\mathrm{n}=3$ & $\mathrm{n}=3$ & \\
\hline Age (years) & $15.6 \pm 2.3$ & $12.3 \pm 2.7$ & 0.25 \\
\hline LH (mIU/mL) & $12.7 \pm 10.0$ & $6.1 \pm 2.9$ & 0.27 \\
\hline FSH (mIU/mL) & $23.7 \pm 22.8$ & $6.6 \pm 5.7$ & 0.08 \\
\hline Basal T (ng/mL) & $4.8 \pm 3.8$ & $4.4 \pm 2.2$ & 0.72 \\
\hline DHT (ng/mL) & $0.1 \pm 0.2$ & $0.09 \pm 0.1$ & 0.22 \\
\hline T/DHT ratio & $49.8 \pm 24.9$ & $57.3 \pm 21.2$ & 0.65 \\
\hline
\end{tabular}

\section{Discussion}

This study documented the clinical, hormonal and genetic features of $46, \mathrm{XY}$ DSD who were considered AIS or $5 \alpha-R D$ according to clinical and hormonal criteria. We summarized clinical, endocrine, and genetic data of 128 Turkish children with 46,XY DSD, collected in only one center with molecular analyses performed in a single laboratory.

In our cohort, $24 \%$ of the $46, \mathrm{XY}$ DSD patients had any variants attributed to disease. This finding is consistent with other studies that reportaround $20-40 \%$ of cases achieve a molecular diagnosis whereas the others remain without diagnosis (24). On the otherhand, lower rates of molecularly diagnosed cases, $16.3 \%$ (11.6\% 5 $\alpha$-RD2, $4.7 \%$ AIS) (25) and $12 \%$ ( $8 \%$ AIS, $4 \% 5 \alpha$-RD2 and $88 \%$ without gene abnormality) (26) have also been reported in some studies. Different studies from Turkey also report different rates of $A R$ or SRD5A2 gene mutationsin Turkish populations $(2,17,27,28)$. One of these studies report their 51 patients with the mutation rates of $A R$ gene $22 \%$ and SRD $5 A 2$ gene $12 \%$ which are similar to our results (2). Recently, in a large cohort of Turkish DSD patients, 143 patients with 46,XY DSD were evaluated and 45 (31.4\%) were genetically proven. In this recent study, the distribution of the molecular diagnosis of $46, X Y$ DSD patients were also presented as $26.6 \%$ SRD5A2, and $22.2 \% A R$ (28). In concordance with most of the literature, our study results showed that the frequency of genetically diagnosed AIS patients were higher than $5 \alpha-R D$ patients in the study sample $(27,29,30,31)$. 


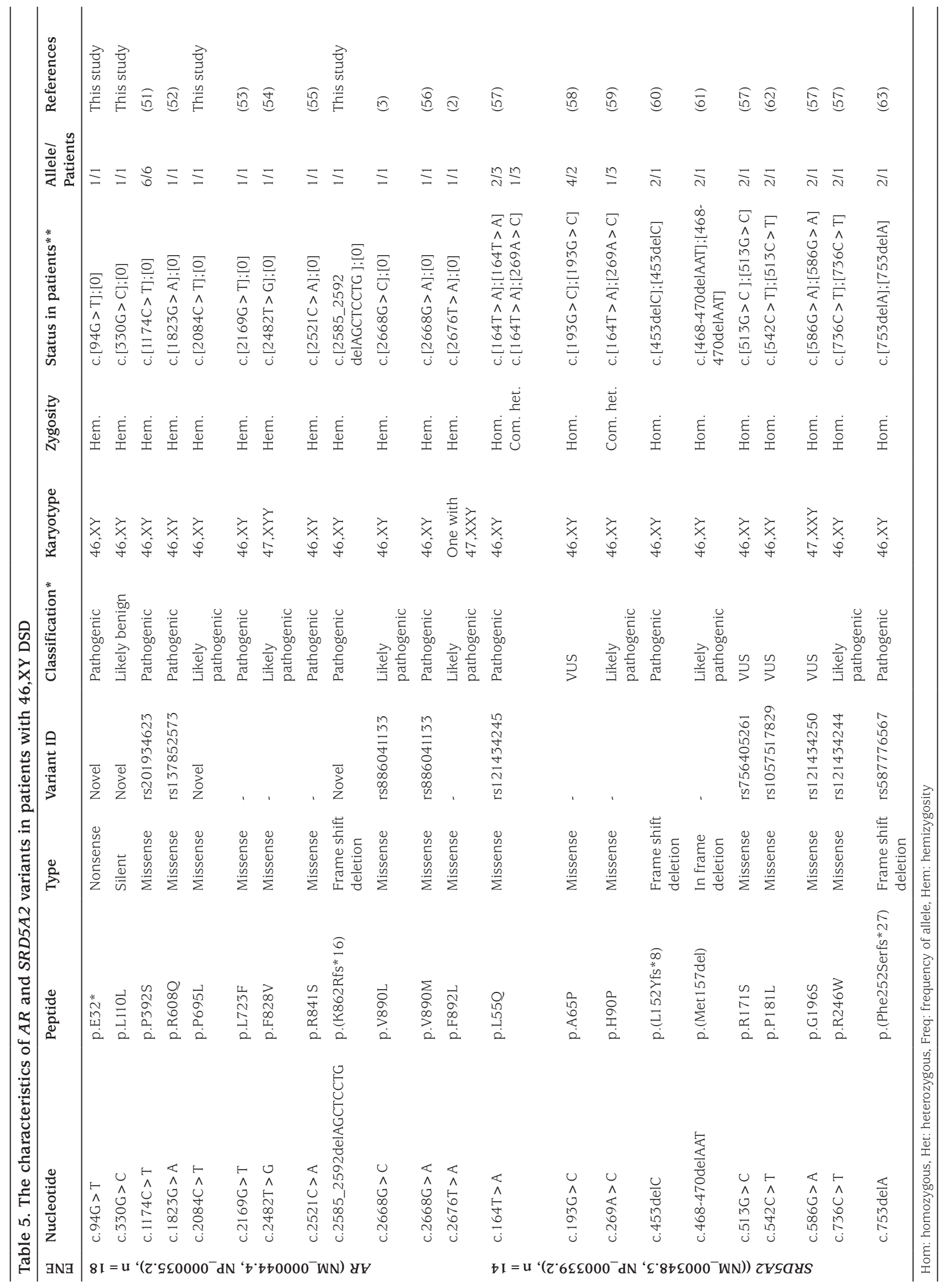


One of the remarkable findings of the current study was the comparison of BW SDS and GA between the subgroups of the study sample. The effect of androgens on fetal growth and BW difference between sexes has been reported in previous studies. Although some studies have shown that BW difference is dependent on fetal androgens, other studies reported that it is not generated by the action of androgens (32). Moreover, it is known that 46,XY DSDs due to nonspecific disorders of undermasculinization are more frequently associated with fetal growth restriction, SGA, and concomitant conditions $(33,34,35,36)$. In the current study, none of the $5 \alpha-$ RD patients had SGA. Moreover, BW SDS and GA of the $5 \alpha-R D$ patients were significantly higher than that of AIS and undiagnosed patients, which may demonstrate that fetal androgens can also affectfetal growth. The $19 \%$ of cases of nonspecific XY DSD without any clear diagnosis is reported to be SGA (16). In this study, $40.4 \%$ of undiagnosed cases was shown to be SGA. The prematurity and intrauterine growth restriction rates were also higher in the undiagnosed group in the current study.Additional conditions are frequent in DSD, with a rate of $27 \%$, which is over 10 times the birth prevalence of congenital anomalies (16). In our study, the rate of other disease comorbidities was higher in the undiagnosed group, in concordance with literature. The presence of one congenital condition may be associated with the presence of further anomalies because disrupting factors, whether environmental or genetic, are likely to affect multiple developmental processes (16).

The different masculinization scores are standardized ways of recording and conveying the degree of virilization on physical examination. These scores are also used to distinguish between individuals with or without any mutation $(37,38)$. Some studies find a correlation between an identifiable genetic cause and masculinization scores whereas some of them do not $(37,38)$. In our study, the CAIS phenotype was more frequent in all ofthe mutation positive groups than genetically undiagnosed patients. Thus, having an identifiable genetic cause may be presented with a lower EMS $(11,12)$ or higher Quigley score $(10)$ compared to patients without an identifiable genetic cause and this trend appears consistent between historical and modern cohorts, despite changes in genetic technology over time.

Different studies use variable cut off levels of T/DHT to differentiate $5 \alpha$-RD from AIS in the laboratory $(1,17,18,27,30-41)$. Cut-off values ranging from 8.5 to 30 have been suggested for the T/DHT ratio (18). The diagnostic interpretations of mean values of T/DHT ratio based on different age groups is still debated. With different sensitivities from different studies, variable mean values of T/DHT in patients with $5 \alpha$-RD during infancy (basal 19.1, peak 29.4), prepuberty (basal 8.0, peak 32.5), adolescence (basal 45.6, peak 71.8) and adulthood (basal 46.6) have been reported (18). In the current study, the same cut-off levels of T/DHT were used for all age groups as a result of missing standardized cut-off levels of ratio according to ages. Lack of precisely determined cut-offs still compromise correct diagnosis, and improperly high or low ratios causes confusion for the reliability of $\mathrm{T} /$ DHT value in clinical practice. Although, AIS cases with T/ DHT $\geq 20$ and $5 \alpha-R D$ cases with T/DHT $<20$ were detected in our study, the significant difference between the mean values of T/DHT ratios between the AIS, $5 \alpha-$ RD and undiagnosed groups may show that this ratio can still be a valuable determinant in laboratory diagnosis. Moreover, the current study demonstrated that when T/DHT was lower than 20, stimulated T/DHT ratio in prepubertal period was significantly lower in AIS than undiagnosed, and higher in $5 \alpha-R D$. Thus, we can speculate that when the ratio is lower than 20 , the lowestvalues may be related to a higher probability of AIS, whereas the higher values, closer to 20, may indicate the probability of being $5 \alpha$ $\mathrm{RD}$. On the other hand, our study found no significance between the basal T/DHT ratio between the subgroups during the minipuberty and puberty. Serum $\mathrm{T}$ and DHT show fluctuations after birth, before declining to normal prepubertal concentrations and these hormones levels also differ according to the Tanner Stage in puberty (1). The ratio is reported to be typically higher in adolescents than infants and pre-pubertal children and a normal range of T/DHT may be 1.5-17 in normal male infants (1). These normal variations may influence the interpretation of basal T/DHT in mini puberty or puberty (1). From this point of view,our study may also lead to new questions about the reliability of this ratio in minipuberty or puberty to differentiate AIS, $5 \alpha-R D$ or undiagnosed group when T/DHT ratio is in the same cut-off range. Also, it may suggest that hCG testing may be more useful in evaluating this ratio.

Although it is not common, 47,XXY, 47,XYY or different karyotypes with $A R$ or SRD5A2 mutations are reported $(42,43)$. In our study the rate of Klinefelter Syndrome was $2.3 \%$ ( $n=3,1$ patient with AIS, 1 patient with $5 \alpha-R D$ and 1 with uncertain diagnosis). Moreover, one patient (0.8\%) with 47,XYY and AR gene mutation was found. Klinefelter patients classically have complete male sex differentiation, and genital anomalies are rarely recognized as associated features of the syndrome (44). The evaluation of $A R$ and SRD5A2 genes in patients with karyotype anomaliesand ambiguous genitalia is also essential to provide accurate genetic counseling for other members of the family. 
Mutations in $A R$ are found in most subjects with CAIS, but the rate has varied between 28-73\%, depending on the case selection $(3,45)$. In our study, $A R$ mutations were found in $63.6 \%$ of patients with clinically completely female phenotype and this rate was consistent with that of some previous studies from Turkey (2). In contrast, SRD5A2 mutations and female external genitalia is reported as rare, $3.9-7.3 \%$ in $5 \alpha$-RD cases $(41,46)$. We described three patients with $S R D 5 A 2$ mutations who had a clinical diagnosis as CAIS (21.4\% of all SRD5A2 patients and $2.3 \%$ of all patients) which was nearly four-fold of the prior reported rate. Interestingly, we also had one patient with clinically CAIS in whom we could not establish a genetic diagnosis and may require whole exome analysis to reveal the diagnosis.

In this research, 12 different variants, four of which were novel, in $A R$ and 10 different variants in SRD5A2 were detected. More than 1000 different mutations in $A R$ leading to AIS have been reported (47). Although exon 1 encodes more than half of the AR protein, exon 1 mutations only represent $25 \%$ of all of the mutations in AIS patients (47). More than $70 \%$ of AIS mutations in exon 1 appear to cause CAIS, and about $18 \%$ of exon 1 mutations are related with MAIS which is due to single-base substitution (47). However, in the current study, most of the $A R$ mutations $(44.4 \%, n=8)$ were located in exon 1 , and none of them presented with CAIS phenotype. All of CAIS patients had AR mutation in the LBD. Also, the most common mutations of the $A R$ gene in AIS are single point mutations that result in an amino acid substitution (45). In parallel to this, in our study thehighest number of mutations were identified as missense type. The c. $1174 \mathrm{C}>\mathrm{T}$ (p.Pro392Ser) variant which was the most frequent pathogenic $A R$ variant in this study, has previously been reported to be related with CAIS, MAIS, PAIS and testicular cancer phenotype, although all of the patients with this mutation presented with PAIS clinically in this study. Compatible with the literature, the c.2169G > T (p.Leu723Phe) variant caused CIAS rather than PAIS, and c.1823G $>$ A (p.Arg608Gln) caused PAIS rather than CAIS (47). Two siblings in our cohort with c.2676T > A (p.Phe892Leu) variant had different clinical phenotypes (one PAIS, one CAIS). Identical $A R$ mutations can lead to variable phenotypic expression because one mutation can produce different phenotypes and appear in different individuals within a family. Despite many studies, it is known that there is no specific correlations between genotypes and phenotypes identified in the AIS patients (6). Forty-five allelic variants that may result in different phenotypes are currently recorded in the McGill AR mutation database. There are no available qualitative data on penetrance at present. The variable phenotypic expression of particular mutations may be due to differences in affected individuals, such as somatic cell embedding (6). On the otherhand, epigenetic repression of AR transcription in mutation-negative AIS (type II) has been studied recently (48). Cofactors can influence AR activity at the transcriptional as well as posttranscriptional level. Methylation-dependent repression of AR mRNA expression can contribute to an incomplete male genital development in a subset of individuals with AIS type II. This epigenetic regulation of AR expression might be established during embryonic development and maintained after differentiation to ensure proper cellular identity (48). The identification of upstream factors responsible for this epigenetic AR mRNA repression will be the next planned step in undiagnosed cases for the future studies.

To date, more than 100 mutations have been described in SRD5A2 gene (Human Gene Mutation Database, http:// www.hgmd.cf.ac.uk/ac/index.php) (46). Itisreported that approximately $60 \%$ are in thehomozygous and $40 \%$ arein compound heterozygousform (46). However, in our study, we have only one compound heterozygous patient $(7.1 \%)$ and highhomozygous rate may be related to the consanguinity rate in the Turkish population. As a consequence, a wide phenotypic range has been described, attributed to the residual enzymatic activity and probably to the individual genetic background without a strong genotype-phenotype correlation in literature (46). One of our frequently found mutations, p. Leu55Gln, which causes decrease in enzymatic activity has only been described in Turkish patientsto date. In parallel to this, in the current study, we describe three patients with this mutation (one compound heterozygous, two homozygous). It is reported that this mutation causes less severe phenotypes, with EMS values ranging from 3.0 to 8.0 , attributable to different residual enzymatic activities caused by different mutations and a genotype-phenotype correlation seems to be the most difficult in this group. In our study, two homozygous mutation-carrying patients presented as PAIS, whereas the compound heterozygous form of this mutation, with a novel p.His90Pro mutation,presented as CAIS clinically. We suggest that more case reports are needed to support our finding based on genotype-phenotype relationship. Pathogenic alterations that interfere with the NADPH binding domain that are within 3-oxo-5-alpha-steroid 4-dehydrogenase domain were also detected in our study (hom, p.Pro181 Leu, hom.p.Gly196Ser, hom. p.Arg246Try, hom. p.Arg171Ser). The EMS scores of these mutations is reported to vary between 2.67 to 4.17 (46). In the current study, homozygous p.Arg246Try alteration was associated witha CAIS phenotype. Although it has been reported that only the p.Gly196Ser variant seems to produce a less variable phenotype (46), allelic variants at exon 4 
and indels consistently have recently been shown to cause more severe phenotypes (49). Variant p.Gly196Ser, identified in homozygous form, is associated witha PAIS phenotype with Quigley score 3 in this study. Another known mutations affecting NADPH domain, p.Arg171Ser, is frequently found in different populations (Mexican, Turkish, Spanish, Mediterranean), there are very few homozygous reported cases, being found more frequently in compound heterozygotes (49). However, we have one homozygous p.Arg171Ser mutant patient who presented with PAIS. Although female appearance genitalia is infrequent in $5 \alpha$-RD cases, we had three CAIS phenotype (one withp. [L55Q];[H90P], one hom. p.Arg246Try, and onewith hom. p.(Leu152Tyrfs*8). Although we have two patients with the same hom. p.(Leu152Tyrfs*8) mutation and one heterozygous form of this mutation in the same family, they presented with different degrees of undermasculization. The genotype-phenotype incongruence occurs even in individuals carrying the same variant and also in individuals from the same family, suggesting that other factors beyond the SRD5A2 enzyme play a role in phenotype (49). Thus, and similar to $A R$ mutations, allelic variants in the SRD5A2 gene, lead to a broad spectrum of external genitalia phenotypes with no strong genotype-phenotype relationship (49) and some other factors that affect phenotype are still unclear for both AR and SRD5A2, and constitute a relevant field for future research.

In our study gonadectomy was performed in 16 patient $(\mathrm{n}=7$ AIS, $\mathrm{n}=5$ SRD5A2 and 4 patient without any identified mutation). Intratubular germ cell neoplasia was only seen in one patient with no any detected mutation in $A R$ or SRD5A2. Indeed, dysgenetic and undescended testes are the major risk factors for testicular cancer, the most common malignancy for men between the ages of 15 and 35 years (50). Our patient with gonadoblastoma was 7.3 years old when the gonadectomy was performed. The other causes of gonadoblastoma in 46XY DSD patients, except for dysgenetic gonads are also reported as AIS, ovotesticular DSD, Klinefelter syndrome, $5 \alpha-R D$, and $17 \beta$-HSD deficiency respectively (50). Unfortunately, we only studied SRD5A2 and $A R$ genes in this patient, and further genetic analysis will be essential to obtain a definitive diagnosis.

\section{Study Limitations}

Our study has some limitations. First, the nature of the study required us to rely on data from medical records. Second, serum levels of inhibin B and anti-Mullerian hormone were not examined due to missing data. Third, according to the wide age range of sample, $\mathrm{T}$ and DHT were measured in different years with a possibility of different methods that may have led to some inaccuracies. These shortcomings can be overcome in future prospective studies by starting to use genetic analysis earlier with more specific methods, such as liquid chromatography linked with tanden mass spectrometry or immunoassays after organic solvent extraction to detect hormones. This study used the Sanger sequencing method for diagnosis of AIS and $5 \alpha-$ RD patients. However, next-generation sequencing-based targeted sequencing is a promising technique to improve the detection rate of DSD, and it will be more useful for future studies.

\section{Conclusion}

Four novel AR variants were identified in our study. T/DHT ratio in the diagnosis of AIS and $5 \alpha-R D$ is an important hormonal criteria, but in some cases, T/DHT ratio may be vary beyond the accepted cut-offs that may lead to diagnostic confusion. So genetic analysis for actual diagnosis seems to be essential, especially for determining the treatment pathway and the sex identity of patients.

\section{Acknowledgement}

We would like to convey special thanks to the Scientific Research Projects Coordination Unit of İstanbul University (project ID TYL-2017-24211).

\section{Ethics}

Ethics Committee Approval: The study was approved by the İstanbul Faculty of Medicine, İstanbul University of Ethics Committee.

Informed Consent: Retrospective study.

Peer-review: Externally and internally peer-reviewed.

\section{Authorship Contributions}

Surgical and Medical Practices - Concept - Design - Data Collection or Processing - Analysis or Interpretation Literature Search - Writing: All authors.

Financial Disclosure: This research was supported by Scientific Research Projects Coordination Unit of Istanbul University (project ID TYL-2017-24211).

\section{References}

1. Pang S, Levine LS, Chow D, Sagiani F, Saenger P, New MI. Dihydrotestosterone and its relationship to testosterone in infancy and childhood. J Clin Endocrinol Metab 1979;48:821-826.

2. Akcay T, Fernandez-Cancio M, Turan S, Güran T, Audi L, Bereket A AR and SRD5A2 gene mutations in a series of 51 Turkish 46,XY DSD children with a clinical diagnosis of androgen insensitivity. Andrology 2014;2:572-578. Epub 2014 Apr 16 
3. Audi L, Fernández-Cancio M, Carrascosa A, Andaluz P, Torán N, Piró C, Vilaró E, Vicens-Calvet E, Gussinyé M, Albisu MA, Yeste D, Clemente M, Hernández de la Calle I, Del Campo M, Vendrell T, Blanco A, MartínezMora J, Granada ML, Salinas I, Forn J, Calaf J, Angerri O, Martínez-Sopena MJ, Del Valle J, García E, Gracia-Bouthelier R, Lapunzina P, Mayayo E, Labarta JI, Lledó G, Sánchez Del Pozo J, Arroyo J, Pérez-Aytes A, Beneyto M, Segura A, Borrás V, Gabau E, Caimarí M, Rodríguez A, Martínez-Aedo MJ, Carrera M, Castaño L, Andrade M, Bermúdez de la Vega JA; Grupo de Apoyo al Sindrome de Insensibilidad a los Andrógenos (GrApSIA). Novel $(60 \%)$ and recurrent $(40 \%)$ androgen receptor gene mutations in a series of 59 patients with a 46,XY disorder of sex development. J Clin Endocrinol Metab 2010;95:1876-1888. Epub 2010 Feb 11

4. Fernández-Cancio M, Audí L, Andaluz P, Torán N, Piró C, Albisu M, Gussinyé M, Yeste D, Clemente M, Martinez-Mora J, Blanco A, Granada ML, Marco M, Ferragut J, López-Siguero JP, Beneyto M, Carles C, Carrascosa A. SRD5A2 gene mutations and polymorphisms in Spanish $46, X Y$ patients with a disorder of sex differentiation. Int J Androl 2011;34:526-535. Epub 2011 Jun 2

5. Batista RL, Costa EMF, Rodrigues AS, Gomes NL, Faria JA Jr, Nishi MY, Arnhold IJP, Domenice S, Mendonca BB. Androgen insensitivity syndrome: a review. Arch Endocrinol Metab 2018;62:227-235.

6. Liu Q, Yin X, Li P. Clinical, hormonal and genetic characteristics of androgen insensitivity syndrome in 39 Chinese patients. Reprod Biol Endocrinol 2020;18:34

7. Ono M, Harley VR. Disorders of sex development: new genes, new concepts. Nat Rev Endocrinol 2013;9:79-91. Epub 2012 Dec 18

8. Carmichael SL, Witte JS, Ma C, Lammer EJ, Shaw GM. Hypospadias and variants in genes related to sex hormone biosynthesis and metabolism. Andrology 2014;2:130-137. Epub 2013 Nov 26

9. Peters M, Saare M, Kaart T, Haller-Kikkatalo K, Lend AK, Punab M, Metspalu A, Salumets A. Analysis of polymorphisms in the SRD5A2 gene and semen parameters in Estonian men. J Androl 2010;31:372378. Epub 2009 Nov 19

10. Quigley CA, De Bellis A, Marschke KB, el-Awady MK, Wilson EM, French FS. Androgen receptor defects: historical, clinical, and molecular perspectives. Endocr Rev 1995;16:271-321.

11. Ahmed SF, Khwaja O, Hughes IA. The role of a clinical score in the assessment of ambiguous genitalia. BJU Int 2000;85:120-124

12. Ahmed SF, Achermann JC, Arlt W, Balen A, Conway G, Edwards Z, Elford S, Hughes IA, Izatt L, Krone N, Miles H, O'Toole S, Perry L, Sanders C, Simmonds M, Watt A, Willis D. Society for Endocrinology UK guidance on the initial evaluation of an infant or an adolescent with a suspected disorder of sex development (Revised 2015). Clin Endocrinol (Oxf) 2016;84:771-788. Epub 2015 Aug 13

13. Neyzi O, Furman A, Bundak R, Gunoz H, Darendeliler F, Bas F. Growth references for Turkish children aged 6 to 18 years. Acta Paediatr 2006; $95: 1635-1641$

14. Telatar B, Comert S, Vitrinel A, Erginöz E. Anthropometric measurements of term neonates from a state hospital in Turkey. East Mediterr Health J 2009;15:1412-1419.

15. Chan YM, Levitsky LL, Baskin LS, Geffner ME, Hoppin AG. Evaluation of the infants with atypical genitalia (disorder of sex development). 2019 UpToDate, www.uptodate.com. Available from: https://www. uptodate.com/contents/evaluation-of-the-infant-with-atypical-genitalappearance-difference-of-sex-development

16. Cox K, Bryce J, Jiang J, Rodie M, Sinnott R, Alkhawari M, Arlt W, Audi L, Balsamo A, Bertelloni S, Cools M, Darendeliler F, Drop S, Ellaithi M, Guran T, Hiort O, Holterhus PM, Hughes I, Krone N, Lisa L, Morel Y, Soder O, Wieacker P, Ahmed SF. Novel associations in disorders of sex development: findings from the I-DSD Registry. J Clin Endocrinol Metab 2014;99:348-355. Epub 2013 Dec 3
17. Topcu V, Ilgin-Ruhi H, Siklar Z, Karabulut HG, Berberoglu M, Hacihamdioglu B, Savas-Erdeve S, Aycan Z, Peltek-Kendirci HN, Ocal G, Tukun FA. Investigation of androgen receptor gene mutations in a series of 21 patients with 46,XY disorders of sex development. J Pediatr Endocrinol Metab 2015;28:1257-1263.

18. Bertelloni S, Russo G, Baroncelli GI. Human Chorionic Gonadotropin Test: Old Uncertainties, New Perspectives, and Value in 46,XY Disorders of Sex Development. Sex Dev 2018;12:41-49. Epub 2017 Nov 2

19. Landrum MJ, Lee JM, Benson M, Brown GR, Chao C, Chitipiralla S, Gu B, Hart J, Hoffman D, Jang W, Karapetyan K, Katz K, Liu C, Maddipatla Z, Malheiro A, McDaniel K, Ovetsky M, Riley G, Zhou G, Holmes JB, Kattman BL, Maglott DR. ClinVar: improving access to variant interpretations and supporting evidence. Nucleic Acids Res 2018;46:1062-1067.

20. Stenson PD, Mort M, Ball EV, Evans K, Hayden M, Heywood S, Hussain M, Phillips AD, Cooper DN. The Human Gene Mutation Database: towards a comprehensive repository of inherited mutation data for medical research, genetic diagnosis and next-generation sequencing studies. Hum Genet 2017;136:665-677. Epub 2017 Mar 27

21. Richards S, Aziz N, Bale S, Bick D, Das S, Gastier-Foster J, Grody WW, Hegde M, Lyon E, Spector E, Voelkerding K, Rehm HL; ACMG Laboratory Quality Assurance Committee. Standards and guidelines for the interpretation of sequence variants: a joint consensus recommendation of the American College of Medical Genetics and Genomics and the Association for Molecular Pathology. Genet Med 2015;17:405-424. Epub 2015 Mar 5

22. Desmet FO, Hamroun D, Lalande M, Collod-Béroud G, Claustres M, Béroud C. Human Splicing Finder: an online bioinformatics tool to predict splicing signals. Nucleic Acids Res 2009;37:e67. Epub 2009 Apr 1

23. Kotlar D, Lavner Y. The action of selection on codon bias in the human genome is related to frequency, complexity, and chronology of amino acids. BMC Genomics 2006;7:67.

24. Baetens D, Mladenov W, Delle Chiaie B, Menten B, Desloovere A, Iotova V, Callewaert B, Van Laecke E, Hoebeke P, De Baere E, Cools M. Extensive clinical, hormonal and genetic screening in a large consecutive series of 46,XY neonates and infants with atypical sexual development. Orphanet J Rare Dis 2014;9:209.

25. Ittiwut C, Pratuangdejkul J, Supornsilchai V, Muensri S, Hiranras Y, Sahakitrungruang T, Watcharasindhu S, Suphapeetiporn K, Shotelersuk $\mathrm{V}$. Novel mutations of the SRD5A2 and AR genes in Thai patients with 46, XY disorders of sex development. J Pediatr Endocrinol Metab 2017;30:19-26.

26. Ahmadifard M, Kajbafzadeh A, Panjeh-Shahi S, Vand-Rajabpour F, Ahmadi-Beni R, Arshadi H, Setoodeh A, Rostami P, Tavakkoly-Bazzaz J, Tabrizi M. Molecular investigation of mutations in androgen receptor and 5-alpha-reductase-2 genes in 46,XY Disorders of Sex Development with normal testicular development. Andrologia 2019;51:e13250. Epub 2019 Feb 27

27. Diaz A, Lipman Diaz EG. Disorders of Sex Development. Pediatr Rev 2021;42:414-426.

28. Ata A, Özen S, Onay H, Uzun S, Gökşen D, Özkınay F, Özbaran NB, Ulman I, Darcan Ş. A large cohort of disorders of sex development and their genetic characteristics: 6 novel mutations in known genes. Eur J Med Genet 2021;64:104154. Epub 2021 Jan 29

29. Délot EC, Papp JC; DSD-TRN Genetics Workgroup, Sandberg DE, Vilain E. Genetics of Disorders of Sex Development: The DSD-TRN Experience. Endocrinol Metab Clin North Am 2017;46:519-537. Epub 2017 Mar 28

30. Joshi RR, Rao S, Desai M. Etiology and clinical profile of ambiguous genitalia an overview of 10 years experience. Indian Pediatr 2006;43:974-979. 
31. Al-Agha AE, Thomsett MJ, Batch JA. The child of uncertain sex: 17 years of experience. J Paediatr Child Health 2001;37:348-351.

32. Poyrazoglu S, Darendeliler F, Ahmed SF, Hughes I, Bryce J, Jiang J, Rodie M, Hiort O, Hannema SE, Bertelloni S, Lisa L, Guran T, Cools M, Desloovere A, Claahsen-van der Grinten HL, Nordenstrom A, Holterhus PM, Kohler B, Niedziela M, Krone N. Birth Weight in Different Etiologies of Disorders of Sex Development. J Clin Endocrinol Metab 2017;102:1044-1050.

33. Ghirri P, Scaramuzzo RT, Bertelloni S, Pardi D, Celandroni A, Cocchi G, Danieli R, De Santis L, Di Stefano MC, Gerola O, Giuffrè M, Gragnani GS, Magnani C, Meossi C, Merusi I, Sabatino G, Tumini S, Corsello G, Boldrini A. Prevalence of hypospadias in Italy according to severity, gestational age and birthweight: an epidemiological study. Ital J Pediatr 2009;35:18

34. Yinon Y, Kingdom JC, Proctor LK, Kelly EN, Salle JL, Wherrett D, Keating S, Nevo O, Chitayat D. Hypospadias in males with intrauterine growth restriction due to placental insufficiency: the placental role in the embryogenesis of male external genitalia. Am J Med Genet A 2010;152A:75-83.

35. Scaramuzzo RT, Boldrini A, Bertelloni S, Parrini D, Serino L, Ghirri P. Low testosterone levels in pre-term newborns born small for gestational age. J Endocrinol Invest 2010;33:215-217. Epub 2010 May 5

36. Huisma F, Thomas M, Armstrong L. Severe hypospadias and its association with maternal-placental factors. Am J Med Genet A 2013;161:2183-2187. Epub 2013 Aug 2

37. Deeb A, Mason C, Lee YS, Hughes IA. Correlation between genotype, phenotype and sex of rearing in 111 patients with partial androgen insensitivity syndrome. Clin Endocrinol (Oxf) 2005;63:56-62.

38. Su R, Adam MP, Ramsdell L, Fechner PY, Shnorhavorian M. Can the external masculinization score predict the success of genetic testing in 46,XY DSD? AIMS Genetics 2015;2:163-172.

39. Veiga-Junior NN, Medaets PA, Petroli RJ, Calais FL, de Mello MP, Castro CC, Guaragna-Filho G, Sewaybricker LE, Marques-de-Faria AP, MacielGuerra AT, Guerra-Junior G. Clinical and Laboratorial Features That May Differentiate 46,XY DSD due to Partial Androgen Insensitivity and 5 $\alpha$-Reductase Type 2 Deficiency. Int J Endocrinol 2012;2012:964876. Epub 2011 Dec 12

40. Cheng J, Lin R, Zhang W, Liu G, Sheng H, Li X, Zhou Z, Mao X, Liu L. Phenotype and molecular characteristics in 45 Chinese children with $5 \alpha$-reductase type 2 deficiency from South China. Clin Endocrinol (Oxf) 2015;83:518-526. Epub 2015 May 7

41. Maimoun L, Philibert P, Cammas B, Audran F, Bouchard P, Fenichel P, Cartigny M, Pienkowski C, Polak M, Skordis N, Mazen I, Ocal G, Berberoglu M, Reynaud R, Baumann C, Cabrol S, Simon D, KayembaKay's K, De Kerdanet M, Kurtz F, Leheup B, Heinrichs C, Tenoutasse S, Van Vliet G, Grüters A, Eunice M, Ammini AC, Hafez M, Hochberg Z, Einaudi S, Al Mawlawi H, Nuñez CJ, Servant N, Lumbroso S, Paris F, Sultan C. Phenotypical, biological, and molecular heterogeneity of $5 \alpha$-reductase deficiency: an extensive international experience of 55 patients. J Clin Endocrinol Metab 2011;96:296-307. Epub 2010 Dec 8

42. Shabir I, Khurana ML, Joseph AA, Eunice M, Mehta M, Ammini AC. Phenotype, genotype and gender identity in a large cohort of patients from India with 5 $\alpha$-reductase 2 deficiency. Andrology 2015;3:1132-1139.

43. Atta I, Ibrahim M, Parkash A, Lone SW, Khan YN, Raza J. Etiological diagnosis of undervirilized male/XY disorder of sex development. J Coll Physicians Surg Pak 2014;24:714-718.

44. Lee YS, Cheng AW, Ahmed SF, Shaw NJ, Hughes IA. Genital anomalies in Klinefelter's syndrome. Horm Res 2007;68:150-155. Epub 2007 Jul 19

45. Jääskeläinen J. Molecular biology of androgen insensitivity. Mol Cell Endocrinol 2012;352:4-12. Epub 2011 Aug 17
46. Avendaño A, Paradisi I, Cammarata-Scalisi F, Callea M. 5- $\alpha$-Reductase type 2 deficiency: is there a genotype-phenotype correlation? A review. Hormones (Athens) 2018;17:197-204. Epub 2018 Apr 20

47. Gottlieb B, Beitel LK, Nadarajah A, Paliouras M, Trifiro M. The androgen receptor gene mutations database: 2012 update. Hum Mutat 2012;33:887-894. Epub 2012 Mar 13

48. Hornig NC, Rodens P, Dörr H, Hubner NC, Kulle AE, Schweikert HU, Welzel M, Bens S, Hiort O, Werner R, Gonzalves S, Eckstein AK, Cools M, Verrijn-Stuart A, Stunnenberg HG, Siebert R, Ammerpohl O, Holterhus PM. Epigenetic Repression of Androgen Receptor Transcription in Mutation-Negative Androgen Insensitivity Syndrome (AIS Type II). J Clin Endocrinol Metab 2018;103:4617-4627.

49. Batista RL, Mendonca BB. Integrative and Analytical Review of the 5-Alpha-Reductase Type 2 Deficiency Worldwide. Appl Clin Genet 2020;13:83-96.

50. Ulbright TM, Young RH. Gonadoblastoma and selected other aspects of gonadal pathology in young patients with disorders of sex development. Semin Diagn Pathol 2014;31:427-440. Epub 2014 Jul 18

51. Bhangoo A, Paris F, Philibert P, Audran F, Ten S, Sultan C. Isolated micropenis reveals partial androgen insensitivity syndrome confirmed by molecular analysis. Asian J Androl 2010;12:561-566.

52. Zhang T, Karsh LI, Nissenblatt MJ, Canfield SE. Androgen Receptor Splice Variant, AR-V7, as a Biomarker of Resistance to Androgen Axis-Targeted Therapies in Advanced Prostate Cancer. Clin Genitourin Cancer 2020;18:1-10. Epub 2019 Sep 26

53. Elfferich P, van Royen ME, van de Wijngaart DJ, Trapman J, Drop SL, van den Akker EL, Lusher SJ, Bosch R, Bunch T, Hughes IA, Houtsmuller AB, Cools M, Faradz SM, Bisschop PH, Bunck MC, Oostdijk W, Brüggenwirth HT, Brinkmann AO. Variable loss of functional activities of androgen receptor mutants in patients with androgen insensitivity syndrome. Sex Dev 2013;7:223-234.

54. Chávez B, Méndez JP, Ulloa-Aguirre A, Larrea F, Vilchis F. Eight novel mutations of the androgen receptor gene in patients with androgen insensitivity syndrome. J Hum Genet. 2001;46:560-565.

55. Melo KF, Latronico AC, Costa EM, Billerbeck AE, Mendonca BB, Arnhold IJ. A novel point mutation (R840S) in the androgen receptor in a Brazilian family with partial androgen insensitivity syndrome. Hum Mutat 1999;14:353.

56. Gottlieb B, Vasiliou DM, Lumbroso R, Beitel LK, Pinsky L, Trifiro MA. Analysis of exon 1 mutations in the androgen receptor gene. Hum Mutat 1999;14:527-539.

57. Thigpen AE, Davis DL, Milatovich A, Mendonca BB, Imperato-McGinley J, Griffin JE, Francke U, Wilson JD, Russell DW. Molecular genetics of steroid 5 alpha-reductase 2 deficiency. J Clin Invest 1992;90:799-809.

58. Savas Erdeve S, Aycan Z, Berberoglu M, Siklar Z, Hacihamdioglu B, Sipahi K, Akar N, Ocal G. A novel mutation of 5alpha-steroid reductase 2 deficiency (CD 65 ALA-PRO) with severe virilization defect in a Turkish family and difficulty in gender assignment. Eur J Pediatr 2010;169:991-995.

59. Gui B, Song Y, Su Z, Luo FH, Chen L, Wang X, Chen R, Yang Y, Wang J, Zhao X, Fan L, Liu X, Wang Y, Chen S, Gong C. New insights into $5 \alpha$-reductase type 2 deficiency based on a multi-centre study: regional distribution and genotype-phenotype profiling of SRD5A2 in 190 Chinese patients. J Med Genet 2019;56:685-692.

60. Di Marco C, Bulotta AL, Varetti C, Dosa L, Michelucci A, Baldinotti F, Meucci D, Castagnini C, Lo Rizzo C, Di Maggio G, Simi P, Mari F, Bertelloni S, Renieri A, Messina M. Ambiguous external genitalia due to defect of 5 - $\alpha$-reductase in seven Iraqi patients: prevalence of a novel mutation. Gene 2013;526:490-493.

61. Boudon C, Lobaccaro JM, Lumbroso S, Ogur G, Ocal G, Belon C, Sultan C. A new deletion of the 5 alpha-reductase type 2 gene in a 
Turkish family with 5 alpha-reductase deficiency. Clin Endocrinol (Oxf) 1995;43:183-188.

62. Nicoletti A, Baldazzi L, Balsamo A, Barp L, Pirazzoli P, Gennari M, Radetti G, Cacciari E, Cicognani A. SRD5A2 gene analysis in an Italian population of under-masculinized 46,XY subjects. Clin Endocrinol (Oxf) 2005;63:375-380.
63. Can S, Zhu YS, Cai LQ, Ling Q, Katz MD, Akgun S, Shackleton $\mathrm{CH}$, Imperato-McGinley J. The identification of 5 alpha-reductase- 2 and 17 beta-hydroxysteroid dehydrogenase-3 gene defects in male pseudohermaphrodites from a Turkish kindred. J Clin Endocrinol Metab 1998;83:560-569.

Click for Supplementary Table 1 and Supplementary Table 2 access link: http://glns.co/9c1 eo 